\title{
Danhong Injection Combined With Naoxintong Capsule in Patients With Acute Coronary Syndrome Patients Undergoing PCl: Study Protocol for a Randomized Controlled Trial
}

\section{hairong cai ( $\sim 286941768 @ q q . c o m$ )}

Guangzhou University of Chinese Medicine

\section{Luo Sicong}

guanghzou university of chinese medicine

\section{Gao Huanjia}

Guangzhou University of Chinese Medicine

\section{Liu Shuling}

Guangzhou University of Traditional Chinese Medicine: Guangzhou University of Chinese Medicine

\section{Zhao Shuai}

guangzhou university of chinese medicine

\section{Li Zhishang}

Guangzhou University of Chinese Medicine

\section{Zhang Weizhang}

Guangzhou University of Chinese Medicine

\section{Tang Yong}

Guangzhou University of Chinese Medicine

\section{Chen Bojun}

Guangzhou University of Chinese Medicine

\section{Study protocol}

Keywords: Acute coronary syndrome, Danhong injection, Naoxintong capsule, randomized controlled trial, protocol

Posted Date: June 29th, 2021

DOl: https://doi.org/10.21203/rs.3.rs-654226/v1

License: (c) (i) This work is licensed under a Creative Commons Attribution 4.0 International License. Read Full License 


\section{Abstract}

\section{Background:}

Acute coronary syndrome (ACS) is a group of clinical syndromes caused by thrombosis caused by rupture of coronary atherosclerotic plaque or erosion ulcer, resulting in complete or incomplete occlusion of blood vessels. ACS is the most common cardiovascular critical disease. Although with the development of coronary intervention technology, the incidence of major adverse cardiovascular events (MACE) is still high. In China, traditional Chinese medicine (TCM) has been widely used in the adjuvant treatment of ACS to improve the symptoms and prognosis. Danhong injection (DHI) and Naoxintong capsule (NXTC) may improve the prognosis of patients with ACS, but the evidence-based evidence is still insufficient. The main purpose of this study was to evaluate the efficacy and safety of DHI combined with NXTC in the treatment of ACS.

\section{Methods/design:}

This is a randomized, placebo-controlled clinical trial.1752 patients with ACS undergoing percutaneous coronary intervention (PCl) will be randomly assigned to receive DHI combined with NXTC or placebo at the ratio of 1:1. The course of treatment was 12 weeks. All participants received conventional treatment. The main outcome measure was the 12-months incidence of MACEs. Adverse events (AE) will also be evaluated.

\section{Discussion:}

This trial is a well designed study according to principles and regulations issued by the China food and Drug Administration (CFDA). The results will provide high-quality evidence for the efficacy and safety of DHI combined with NXTC in the treatment of ACS. The results of this study can provide clinicians with evidence-based recommendations for treatment of ACS.

Trial registration冈Chinese Clinical Trial Registry(http://www.chictr.org.cn/index.aspx), ChiCTR-IOR14005693.Registered on November 12,2014.

\section{Introduction}

\section{Background and rationale}

Acute coronary syndrome (ACS) refer to the array of clinical signs and symptoms produced by acute myocardial ischemia generally result from the atherosclerotic plaque rupture or chronic stenosis, includes ST-segment elevation myocardial infarction (STEMI), non-ST-segment elevation myocardial infarction (NSTEMI), and unstable angina (UA) [1-4]. With the development of social economy and the continuous change of life style, the morbidity rates of ACS in China also present a rising trend [5-6]. ACS is one of the main leading cause of death which seriously threatens the health and quality of human life in China [7-8]. The Global Registry of Acute Coronary Events (GRACE) found that the 1-year case-mortality rate of 
ACS was about $15 \%$ and the 5-year cumulative incidence of mortality was as high as $20 \%$ [9-10]. According to the 5-year follow-up results of 3670 AMI patients reported by Bougouin [11], the in-hospital mortality rate was $5.6 \%$ and 5 -year all-cause mortality among patients surviving to be discharged ranged up to $26 \%$. According to the American Heart Association [12], the mortality of cardiovascular diseases were reduced from 295.7 per 100,000 individuals in 2004 to 222.3 per 100,000 individuals in 2014 in Unite States. The EPICOR Asia study [13] also pointed out that the total 1-year incidence rate of coronary artery events among STEMI patients discharge from hospital in Asia was $12.5 \%$, with a mortality rate of up to $3.4 \%$.Taiwan acute coronary syndrome full spectrum registry [14] found that 1-year case-mortality rate after onset of STEMI, NSTEMI and UA was $6.1 \%, 10.1 \%$ and $6.2 \%$ among the 3183 ACS patients, respectively. In terms of chronic coronary disease (CAD), ACS typically means worse prognosis, heavier treatment burden, and higher risk of death. Although the mortality of ACS has decreased with advances in percutaneous coronary intervention (PCl) and pharmacologic therapies, the incidence of major adverse cardiovascular events (MACE) is still high [15].

The integration of Chinese and Western medicine is the most well characterized methods in the treatment of ACS in China, which improve quality of life and symptoms such as angina pectoris and palpitations [16-17]. DHI and NXTC are the main Chinese medicines in the treatment of ACS in China. Various studies indicated that DHI and NXTC have various pharmacological functions including improvement in endothelial action, anti-inflammatory, antioxidant properties, and antithrombotic effects [18-22]. Clinical studies found that DHI and NXTC can have the effects of regulating blood glucose, regulating blood lipids, antiplatelet, anticoagulation, improving heart function, improving endothelial function, improving symptoms, improving quality of life in patients with ACS [23-24]. However, due to the small sample size and unscientific research methods, the conclusions are limited. Moreover, previous studies did not observe the effect of DHI combined with NXTC on the incidence of MACEs in patients with ACS. Therefore, we aim to conduct a randomized controlled clinical trial (RCT) to evaluate the efficacy and safety of DHI combined with NXTC in the treatment of ACS.

\section{Objectives}

The objective of this study is to evaluate the efficacy and safety of Danhong injection combined with Naoxintong Capsule in the treatment of patients with acute coronary syndrome undergoing Percutaneous Coronary intervention (PCl).

\section{Trial design}

This is a randomized, placebo-controlled clinical trial (Fig.1). The study was conducted in Guangdong Provincial Hospital of Traditional Chinese Medicine (the Second Affiliated Hospital of Guangzhou University of Chinese Medicine) and will be conducted in accordance with the principles of the Helsinki Declaration (Edinburgh, 2000 EDITION).

The study will continue for eight years. Patients who met the diagnostic criteria for ACS will be assigned to the treatment group or placebo group in a 1:1 ratio. The researchers will conduct screening based on 
established criteria and pre standard treatment plans. Data collection will start with basic data collection until the end of follow-up work (Table 1). The researchers will conduct screening in accordance with the established criteria and pre-standard treatment plan. Data collection will start from the collection of basic data until the end of follow-up (Table 1).

\section{Methods: Participants, Interventions Andoutcomes}

The protocol for this trial is reported based on the Standard Protocol Items: Recommendations for inter ventional trials (spirit) 2013 checklist: defining standard protocol items for clinical trials (additional file 1). The study has been approved by the ethics committee of Guangdong Provincial Hospital of Traditional Chinese Medicine (the Second Affiliated Hospital of Guangzhou University of Chinese Medicine) (approval number YF2014-124-16) and has been registered in the Chinese Clinical Trial Registry (Registration number ChiCTR-IOR-14005693). This study is still in process.

Study setting

This trial will be conducted at the Guangdong Provincial Hospital of Chinese Medicine (GPHCM) in Guangzhou, Guangdong Province, China.

The GPHCM, a 300-bed hospital, is the largest hospital of Chinese Medicine in China. The annual amount of $\mathrm{PCl}$ in GPHCM G was more than 1000 per year.

Eligibility criteria

Inclusion criteria

1.The patients met the diagnostic criteria of acute coronary syndrome [1] and undergoing PCl;

2.The age ranged from 18 to 65 years old;

3.They agreed to participate in the study and signed the informed consent.

Exclusion criteria

1.Severe arrhythmia: III degree atrioventricular block, sick sinus syndrome;

2.Patients after cardiac arrest or cardiopulmonary resuscitation;

3.Severe heart failure: New York heart function class IV;

4.Acute cerebrovascular disease (ischemic stroke, hemorrhagic stroke);

5.Severe blood system diseases (hemophilia, severe anemia, thrombocytopenic purpura, essential thrombocythemia); 
6.Severe renal insufficiency (serum creatinine level $\geq 265 \mathrm{umol} / \mathrm{L}$ );

7.Severe liver disease (cirrhosis or liver cancer), liver dysfunction (serum alanine aminotransferase/aspartate aminotransferase $>3$ times the upper limit of normal);

8.Those who may affect drug absorption after gastrointestinal surgery;

9.Malignant tumor or life expectancy $\leq 2$ years;

10.People with mental disorders or mental retardation;

11.Patients requiring coronary artery bypass grafting (heart bypass);

12.Allergy to the known ingredients of the test drug;

13.Severe respiratory diseases such as chronic obstructive pulmonary disease, moderate to severe pulmonary hypertension, pulmonary embolism.

14.Women who are breast-feeding or pregnant, or women of childbearing age who plan to become pregnant within 6 months of the screening examination, or those who have a positive urine pregnancy test; or male patients who plan to give birth or sperm donation;

15.Suspected or true history of alcohol or drug abuse;

16.Patients who have participated in clinical trials of other drugs within 3 months before screening;

17.Other patients who the investigator considers inappropriate to participate in this study.

Discharge criteria

Discharge criteria are as follows:

1.requirement for individuals to withdraw during the test;

2.violation of the test program;

3.occurrence of serious adverse events (AEs).

Withdrawal or dropout criteria

Patients will not be included in the analysis if:

1.After randomization, the researchers found that the participants were misdiagnosed as acute coronary syndrome.

2.Participants did not receive medication according to the study plan. 
3.The lack of necessary data affected the evaluation of main outcome indicators.

4.The use of prohibited drugs or combined use of drugs dose beyond the provisions of the agreement, thus affecting the evaluation of curative effect.

5.Due to complications or changes in the condition, other specific treatment measures are not suitable for this study to continue.

\section{The patient withdraw.}

Who will take informed consent?

The researchers will provide information about the study and obtain oral and written informed consent.

Additional consent provisions for collection and use of participant data and biological specimens

Additional consent for collection and use of their human biological specimens will be obtained before they are enrolled in the study. Consent for publication Written informed consent will be obtained for use of data for publication from each participant.

Interventions

Explanation for the choice of comparators

Standard anti-platelet, anticoagulation, lipid-lowering medication, and beta blocker therapy are based on recommended guidelines for ACS.

Intervention description

All the patients undergoing $\mathrm{PCl}$ in the two groups will received standard anti-platelet, anticoagulation, lipid-lowering medication, and beta blocker therapy if tolerate in accordance with the practice guidelines for management of patients with ACS. Patients with diabetes or hypertension were treated with hypoglycemic and antihypertensive therapy. All the eligible patients will be randomly allocated to the treatment group [ $20 \mathrm{ml} /$ day DHI dissolved in saline for 1 week and Buchang NXTC (1.2mg p.o.3 times a day) for 12 weeks] or the control group [the same volume of intravenous saline infusion for 1 week and placebo capsule (1.2mg p.o.3 times a day) for 12 weeks)]. DHI, NXTC and NXTC placebo were provided by Shanxi Buchang Pharmaceutical Co., Ltd., in China.

Criteria for discontinuing or modifying allocated interventions

1.Serious AEs occurred with the study drug.

2.The disease worsens and needs urgent treatment.

3. Termination of research by administrative department. 
Strategies to improve adherence to interventions

In order to improve adherence to intervention protocols, we will use pill count economic compensation, free laboratory examination and free treatment.

Participants will be given USD 100 for completing the 3-month outcome assessment. All treatments, all laboratory tests and echocardiography are free for participants. At enrollment, participants are given a variety of items designed to help promote enthusiasm for the project including nominal gifts such as a thermos cup, a pedometer.

Relevant concomitant care permitted or prohibited during the trial

Participants were prohibited from taking other traditional Chinese medicines.

Provisions for post-trial care

If the patient has an adverse reaction related to the trial drug, he will receive free treatment and financial compensation

\section{Outcomes}

Primary outcome

The primary end point is the incidence of MACEs, defined as a composite of nonfatal myocardial infarction, nonfatal stroke, and/or death from cardiovascular causes. A 12-month follow-up visit will be conducted for each patient in the clinical office. An independent endpoint adjudication committee reviewed and adjudicated the primary outcome.

Secondary outcomes

1.SAQ: SAQ will be used to evaluate the burden of symptoms, which consist of 5 functional status, including physical limitation, angina stability, angina frequency, treatment satisfaction and disease perception.

2. Laboratory examinations: TC, TG, HAL-C, LDL-C, FBG, SBP, DBP, BNP, cTnl.

3.Echocardiography: Echocardiography will be performed by the ultrasound specialist in the ultrasound room of Guangdong Provincial Hospital of Traditional Chinese Medicine. The EF, FS, LVDs, LVDd, CO and LVW will be measured.

4. NYHA classification: The NYHA classification will be used to grade the symptoms of patients with HF, which is classified into four stages: Class I, II, III, IV.

Safety assessment 
The safety assessment will be performed for every participant. The individuals' vital signs and general physical status will be examined at every visit. The occurrence of AEs will be checked at visits 2,3,4. All AEs should be documented in the case report form including occurrence time, severity, duration, adopted measure, and the outcome of the $A E$, whether or not they are related to therapy. All AEs will be evaluated for causal relationships.

Participant timeline

For participants randomized to the treatment or control group, study participation will continue for 12 weeks. A total of 4 visits are foreseen: at baseline, 2 interim visits after 4, 8,and 12 weeks and 6 months, and a final visit after 12 monthss (Tab. 1).

Sample size calculation

Previous studies have shown that the incidence of MACEs in patients with ACS 12 months after PCI was $16 \%$, while the expected reduction in the treatment group was $5 \%$. Based on a two-sided test size of a level of $5 \%$ and a power of $80 \%$, a minimum of 730 patients were required for each group. Considering the loss-to-follow-up of approximately $20 \%$, a total sample size of 876 patients in each group is needed.

The annual amount of $\mathrm{PCl}$ in Guangdong Provincial Hospital of Traditional Chinese Medicine was more than 1000 per year. we anticipate including 200-300 patients with ACS undergoing PCl a year in this study. Consequently, this study period is set at 8 years.

Study participants and recruitment

We will recruit 1752 patients with ACS undergoing PCI. These participants will be recruited from Guangdong Provincial Hospital of Traditional Chinese Medicine after they meet the inclusion criteria and agree to participate in the study and sign the informed consent form. We have recruited the first patient on January 1, 2015 and recruitment is predicted to end on December 30, 2022. All participants will sign the informed consent form to participate in the clinical trial.

Assignment of interventions: allocation

Sequence generation

Participants will be randomly assigned to the treatment group and the control group at a ratio of 1:1 according to the random number table generated by SPSS 26.0 software package (SPSS Inc. Chicago, Illinois, USA).

Concealment mechanism

The serial numbers assigned to each patient are kept in a duplicate, sealed, numbered envelopes, which will guarantee that both investigators, enrolling staff and patients do not know the grouping situations. 
Implementation

Randomisation sequences will be generated by a statistician at the EvidenceBased Medicine Center of Guangzhou University of Chinese Medicine, who is not part of the trial statistical team. The principal investigators and enrolling study staff are unaware of the sequence.

Assignment of interventions: Blinding

Who will be blinded

This is a double-blinded, parallel-group, superiority, single-center, randomized controlled clinical trial. The observers of outcomes and the participants will be blinded. The research nurses dispensing the medication will know the treatment allocation, however, they will not be involved in data collection and statistical analysis. Allocation shall proceed via duplicate, sealed, numbered envelopes, which will be stored by the clinical trial quality control inspectors and the main person in charge of this research group. The blind bottom shall not be opened without reason during the trial. The outcome assessors and the statisticians will not participate in the treatment, they will perform the outcome evaluation and the statistical analysis independently.

Procedure for unblinding if needed

If patients condition deteriorates or severe adverse reactions occur, emergency unblinding will be allowed.

Data collection and management

Plans for assessment and collection of outcomes

Statistical professionals are responsible for developing statistical analysis plans through consultation with key researchers. The investigators are responsible for data collection and the data including baseline questionnaires, SAQ, clinical outcomes, Laboratory examinations, NYHA classification, Echocardiography and adherence will be prospectively collected throughout the study period according to the participant timeline (Fig.1).

Plans to promote participant retention and complete follow-up

Financial compensation, 4 weeks all free treatments and 5 times free examinations will be carried out to promote participant retention and complete follow-up. At the 12-week assessments, text messages and/or phone calls are sent to all participants to facilitate the outcome assessment scheduling with a reminder text two- and 1 day before the appointment.

Data management 
Both the electronic medical record and EDC system (Electronic Data Capture System) according to the paper CRF will be used to manage data. Every individual participant data will be registered in the medical records/case report form (CRF) and doubly entered into a password-protected, validated, encrypted electronic CRF to improve the data quality by the investigators.

Two full-time graduate students in charge of data monitoring and validation

does not participate in the in the intervention or data collection. The database will be locked under the orders of the Principal Investigator.

The EDC system will be locked once the study and quality assurance procedures are completed.

Confidentiality

All study data about every participant is confidential and no identifying information will be revealed. The password-protected, validated, encrypted electronic CRF will designed to securely store identifiable information.

Plans for collection, laboratory evaluation and storage of biological specimens for genetic or molecular analysis in this trial/future use

This study has no plans for collection or storage of biological specimens for genetic or molecular analysis.

Statistical methods

Statistical methods for primary and secondary outcomes

The analysis will be performed in accordance with the intention to treat principle in this study. The Kolmogorov-Smirnov test will be used to explore data distribution normality. When the normality of the distribution of variables was acceptable, continuous variables will be presented by mean \pm SD and independent-samples t-test will be used. If not, median with interquartile range will be presented and Mann-Whitney $U$ test will be used. Chi-square or Fisher's exact tests will be used for comparison of dichotomous data. The MACE-free survival will be presented using Kaplan-Meier curves.

For all analyses, a two-sided significance level of 0.05 was considered statistically significant. The statistical analysis will be performed by a

statistics expert from the Evidence-Based Medicine Center of Guangzhou University of Chinese Medicine in a blind manner using SPSS 26.0 software package (SPSS Inc. Chicago, Illinois, USA).

Methods for any additional analyses

No subgroup and adjusted analyses are planned. 
Methods in analysis to handle protocol non-adherence and any statistical methods to handle missing data

Sensitivity analyses will be conducted to handle protocol non-adherence and

statistical methods will be used to adjust for missingness of the data and to smooth the data.

Plans to give access to the full protocol, participant level-data and statistical code

There are no plans for granting public access to the full protocol, participant-level dataset, and statistical code.

Oversight and monitoring

Composition of the coordinating center and trial steering committee

Steering Committee is formed by the chairman and representative(s) from the Clinical Research Organization, whose duty is responsible for all final decisions regarding clinical trial/investigation modifications. The coordinating centre is responsible for non-clinical work, including the establishment and management of the researcher folder, the filling of project-related forms, the communication and coordination of the researcher, the sponsor, ethics, and the organization, the initiation of the project, drug management, and SAE reporting, cooperating with supervision and inspection, and receiving researchrelated training. The Scientific Research Council will have access to the final test data set. At the end of the study, the original data and results will be submitted to the Scientific Research Council. Endpoint adjudication committee consists of three cardiologists responsible for diagnosis of major adverse cardiovascular events. A data safety monitoring board (DSMB) will be composed of three medical doctors specialized in Pharmacology and Cardiology. The DSMB is responsible for making recommendations to the sponsor to continue, modify, suspend or terminate the research.

Composition of the data monitoring committee, its role and reporting structure

The Data Monitoring Committee consists of a doctor, a scientific research manager and a statistician. While doctor is responsible for data collection, data classification, storage and statistician for statistical analysis independently.

Description of any interim analyses and stopping guidelines

If SAEs occurs or the efficacy is not as expected or the quality of the trial drug has problems, the ethics committee, the sponsor or the State Food and Drug Administration have the right to terminate the trial. Interim data will be reported to the independent medical monitor according to the charter approximately every 24 months during active recruitment.

Adverse event reporting and harms 
An adverse event (AE) is any unfavorable and unintended sign, symptom, or disease. All AEs should be documented in the case report form including occurrence time, severity, duration, adopted measure, and the outcome of the AE, whether or not they are related to therapy. All AEs will be evaluated for causal relationships which are graded as not related, probably not related, possibly related, probably related or definitely related. Severe adverse event (SAE) were defined as Grade 3-5.

All AEs and SAEs will be reported to the Ethics Committee of GPHCM, the Data Safety Monitoring Committee (DSMC), the national health authorities and the chief of research.

Frequency and plans for auditing trial conduct

The investigators will conduct a daily audit of screening, enrollment, and data review. Sponsor will perform an audit at any time.

Plans for communicating important protocol amendments to relevant parties

Any changes to the research protocol need to be re-approved by the ethics committee of GPHCM and inform the investigators, participants, data supervision committee.

Dissemination plans

We plan to share the study results through presentations at scientific meetings.

Results information will be submitted to Chinese Clinical Trial Registry no later than 1 year after the end of this study.

Authorship eligibility guidelines and any intended use of professionalwriters

No professional writers are planned.

\section{Discussion}

The patients with ACS undergoing PCI still have a high incidence of MACEs. The 1-year incidence of MACEs was as high as $16.3 \%$, which is a serious threat to human health and the quality of life. TCM still serves the Chinese population today as a valuable treatment [25]

. Nowadays, almost all Chinese Medicine doctors choose TCM as an auxiliary therapy for ACS. DHI combined with NXTC are the most promising TCM for ACS. Although it has been reported that DHI combined with NXTC could improve endothelial function in patients with ACS undergoing PCI [23], there are few studies on MACEs. Therefore, we conducted this trial to examine the efficacy and safety of DHI combined with NXTC for ACS. The quality of RCTs of TCM is generally poor. Problems include poor research design and methodology, lack of training and participants for researchers. In order to ensure the quality of the study and verify the conclusion, quality control has been strictly implemented in experimental design and research implementation. The Consolidated Standards of Reporting Trials [26] 
for TCM has been used in the planning research design. A training session will be held for each site to explain the study protocol.

However, There are several limitations in this study. First, since the study is

conducted in Guangzhou, China, it is uncertain that the relative effects of the trial drug will be similar in other regions or ethnic groups. Second, the follow-up time is relatively short.

Trial status

The study is still ongoing, with first participant enrolled on January 1, 2015 and recruitment is predicted to end on December 30, 2022. The first version was developed in June 20, 2014. Shown above is the third version whose protocol was revised for the following reasons: change of primary outcome, imprecise sample size calculation. To date, 600 participants have been recruited.

\section{Abbreviations}

EF: ejection fraction; FS: fractional shortening; LVDs : left ventricular internal dimension in systole; LVDd: left ventricular internal dimension in diastole; CO: cardiac output; LVW : left ventricular weight; ACS: Acute coronary syndrome; MACE: major adverse cardiovascular event; TCM: traditional Chinese medicine; DHI: Danhong injection; NXTC: Naoxintong capsule; PCl: percutaneous coronary intervention; AE: Adverse events; STEMI: ST-segment elevation myocardial infarction (STEMI); NSTEMI: non-ST-segment elevation myocardial infarction; UA : unstable angina (UA); GRACE: Global Registry of Acute Coronary Events; CAD: chronic coronary disease; GPHCM: Guangdong Provincial Hospital of Chinese Medicine; DSMB: data safety monitoring board; DSMC: Data Safety Monitoring Committee; SAE: Severe adverse event; TC: Total cholesterol; TG: Triglycerides; HDL-C: High density lipoprotein cholesterol; LDL-C: Low density lipoprotein cholesterol; FBG: Fasting blood glucose; SBP: Systolic blood pressure; DBP: Diastolic blood pressure; BNP: Brain natriuretic peptide; cTnl: cTroponin I.

\section{Declarations}

Dissemination policy

Results will be published in peer-reviewed journals and presented at national and international scientific meetings regardless of the magnitude or direction of effect.

Ethics approval and consent to participate

This trial has been approved by the Ethics Committee of the Second Affiliated Hospital of Guangzhou University of Chinese Medicine (YF2014-168-12) and has been registered in China Clinical Registration Center (ChiCTR-IOR-14005693). All subjects will receive written informed consent prior to enrollment.

Consent for publication

Page 13/19 
Not applicable. There is no individual patient data in this manuscript.

Availability of data and materials

Not applicable. All data are fully available without restriction.

Competing interests

The authors declare that they have no competing interests.

Funding

The study was supported by Shanxi Buchang Pharmaceutical Co., Ltd., in China. The funding body did not have any role in the study design, data collection, or the writing of the manuscript, or decision to submit the manuscript for publication.

Name and contact information for the trial sponsor

Shanxi Buchang Pharmaceutical Co., Ltd.

Address: 16 Buchang Road, Qindu District, Xianyang City, Shaanxi Province, China

Telephone: 020-39318108

Email:kefuzhongxin@buchang.com

Authors'contributions

CHR conceived and designed this prospective trial and drafted the manuscript. LSC helped design the prospective trial, drafted the manuscript and participated in data collection. GHJ helped draft the manuscript. LSL helped modify this prospective trial. ZS determined the statistical analysis plan. LZS calculated the sample size and participated in statistical analysis. ZWZ helps with data collection and data management ZJQ was originally Responsible for the quality control of the test. The registration was done by TY on the websites of Chinese clinical trial registry. CBJ is involved in quality control. All authors have read and approved the final manuscript.

Acknowledgements

Thanks to Yan-hong Chen of Guangzhou University of Chinese Medicine for her help.

Authors' information

${ }^{1}$ The Second Clinical Medical School, Guangzhou University of Chinese Medicine, Guangzhou City 510006, Guangdong Province, China. 
${ }^{2}$ The Second Affiliated Hospital, Guangzhou University of Chinese Medicine,

Guangzhou 510006, Guangdong Province, China.

${ }^{3}$ The Third Affiliated Hospital, Guangzhou University of Chinese Medicine,

Guangzhou 510240, Guangdong Province, China.

\section{References}

1. Collet JP, Thiele H, Barbato E, et al. 2020 ESC Guidelines for the management of acute coronary syndromes in patients presenting without persistent ST-segment elevation. Eur Heart J. 2020 Aug 29:ehaa575.

2. Kimura K, Kimura T, Ishihara M, Nakagawa Y, et al. JCS 2018 Guideline on Diagnosis and Treatment of Acute Coronary Syndrome. Circ J. 2019 Apr 25;83(5):1085-1196.

3. Amsterdam EA, Wenger NK, Brindis RG, et al, ACC/AHA Task Force Members. 2014 AHA/ACC guideline for the management of patients with non-ST-elevation acute coronary syndromes: a report of the American College of Cardiology/American Heart Association Task Force on Practice Guidelines. Circulation. 2014 Dec;23(25):e344-426. 130(.

4. Chew DP, Scott IA, Cullen L, et al. National Heart Foundation of Australia and Cardiac Society of Australia and New Zealand: Australian clinical guidelines for the management of acute coronary syndromes 2016. Med J Aust. 2016 Aug 1;205(3):128 - 33.

5. Hayes SN, Kim ESH, Saw J, et al. Spontaneous Coronary Artery Dissection: Current State of the Science: A Scientific Statement From the American Heart Association. Circulation. 2018 May 8;137(19):e523-e557.

6. Zhao E, Lowres N, Woolaston A, et al. Prevalence and patterns of cognitive impairment in acute coronary syndrome patients: A systematic review. Eur J Prev Cardiol. 2020 Feb;27(3):284-93.

7. Zhou M, Liu J, Hao Y, et al. Prevalence and in-hospital outcomes of diabetes among patients with acute coronary syndrome in China: findings from the Improving Care for Cardiovascular Disease in China-Acute Coronary Syndrome Project. Cardiovasc Diabetol. 2018 Nov 27;17(1):147.

8. Peng Y, Du X, Rogers KD, et al. Clinical Pathways for Acute Coronary Syndromes in China (CPACS) Investigators. Predicting In-Hospital Mortality in Patients With Acute Coronary Syndrome in China. Am J Cardiol. 2017 Oct 1;120(7):1077-1083.

9. Fox KA, Anderson FA Jr, Goodman SG, et al. Time course of events in acute coronary syndromes: implications for clinical practice from the GRACE registry. Nat Clin Pract Cardiovasc Med. 2008 Sep;5(9):580-9.

10. ox KA, Carruthers KF, Dunbar DR, et al. Underestimated and under-recognized: the late consequences of acute coronary syndrome (GRACE UK-Belgian Study). Eur Heart J. 2010 Nov;31(22):2755-64. 
11. Bougouin W, Marijon E, Puymirat E, et al. Incidence of sudden cardiac death after ventricular fibrillation complicating acute myocardial infarction: a 5-year cause-of-death analysis of the FAST-MI 2005 registry. Eur Heart J. 2014 Jan;35(2):116-22.

12. Benjamin EJ, Blaha MJ, Chiuve SE, et al. Heart Disease and Stroke Statistics-2017 Update: A Report From the American Heart Association. Circulation. 2017 Mar;7(10):e146-603. 135(.

13. Huo Y, Lee SW, Sawhney JPS, et al. Two-year outcomes post-discharge in Asian patients with acute coronary syndrome: Findings from the EPICOR Asia study. Int J Cardiol. 2020 Sep 15;315:1-8.

14. Chu CY, Lin TH, Lai WT. The Management and Prognostic Factors of Acute Coronary Syndrome: Evidence from the Taiwan Acute Coronary Syndrome Full Spectrum Registry. Acta Cardiol Sin. 2017 Jul;33(4):329-38.

15. Berwanger O, Santucci EV, de Barros E, Silva PGM, et al. Effect of Loading Dose of Atorvastatin Prior to Planned Percutaneous Coronary Intervention on Major Adverse Cardiovascular Events in Acute Coronary Syndrome: The SECURE-PCI Randomized Clinical Trial. JAMA. 2018 Apr;3(13):1331-40. 319.

16. Zhang DW, Wang SL, Wang PL, et al. The efficacy of Chinese herbal medicines on acute coronary syndrome with renal insufficiency after percutaneous coronary intervention. J Ethnopharmacol. 2020 Feb;10:248:112354.

17. Zhou Z, Shen W, Yu L, et al. A Chinese patent medicine, Shexiang Baoxin Pill, for Non-ST-elevation acute coronary syndromes: A systematic review. J Ethnopharmacol. 2016 Dec 24;194:1130-1139.

18. Wang Z, Shi H, Zhao H, et al. Naoxintong Retards Atherosclerosis by Inhibiting Foam Cell Formation Through Activating Ppara Pathway. Curr Mol Med. 2018;18(10):698-710.

19. Yang S, Liu M, Chen Y, et al. NaoXinTong Capsules inhibit the development of diabetic nephropathy in db/db mice. Sci Rep. 2018 Jun;14(1):9158. 8(.

20. Chen L, Zhang L, Fang Z, et al. Naoxintong restores collateral blood flow in a murine model of hindlimb ischemia through PPARס-dependent mechanism. J Ethnopharmacol. 2018 Dec 5;227:121130.

21. Feng $X, L i Y$, Wang $Y$, et al. Danhong injection in cardiovascular and cerebrovascular diseases: Pharmacological actions, molecular mechanisms, and therapeutic potential. Pharmacol Res. 2019 Jan;139:62-75.

22. Lyu M, Yan CL, Liu HX, et al. Network pharmacology exploration reveals endothelial inflammation as a common mechanism for stroke and coronary artery disease treatment of Danhong injection. Sci Rep. 2017 Nov 13;7(1):15427.

23. Lv Y, Pan Y, Gao Y, et al. Effect of Danhong Injection Combined with Naoxintong Tablets on Prognosis and Inflammatory Factor Expression in Acute Coronary Syndrome Patients Undergoing Percutaneous Coronary Intervention. Acta Cardiol Sin. 2015 Jul;31(4):301-7.

24. Zhao S, Tang Y, Cai H, et al. Treatment of Danhong Injection Combined with Naoxintong Capsule in Acute Coronary Syndrome Patients Undergoing PCI Operation: Study for a Randomized Controlled and Double-Blind Trial. Evid Based Complement Alternat Med. 2018 Mar;7;2018:8485472. 
25. Wang J, Wong YK, Liao F. What has traditional Chinese medicine delivered for modern medicine? Expert Rev Mol Med. 2018 May;11:20:e4.

26. Hemming K, Taljaard M, McKenzie JE, et al. Reporting of stepped wedge cluster randomised trials: extension of the CONSORT 2010 statement with explanation and elaboration. BMJ. 2018;363:k1614. Nov 9;.

\section{Tables}

Table 1 Measurement items and points of data capture 


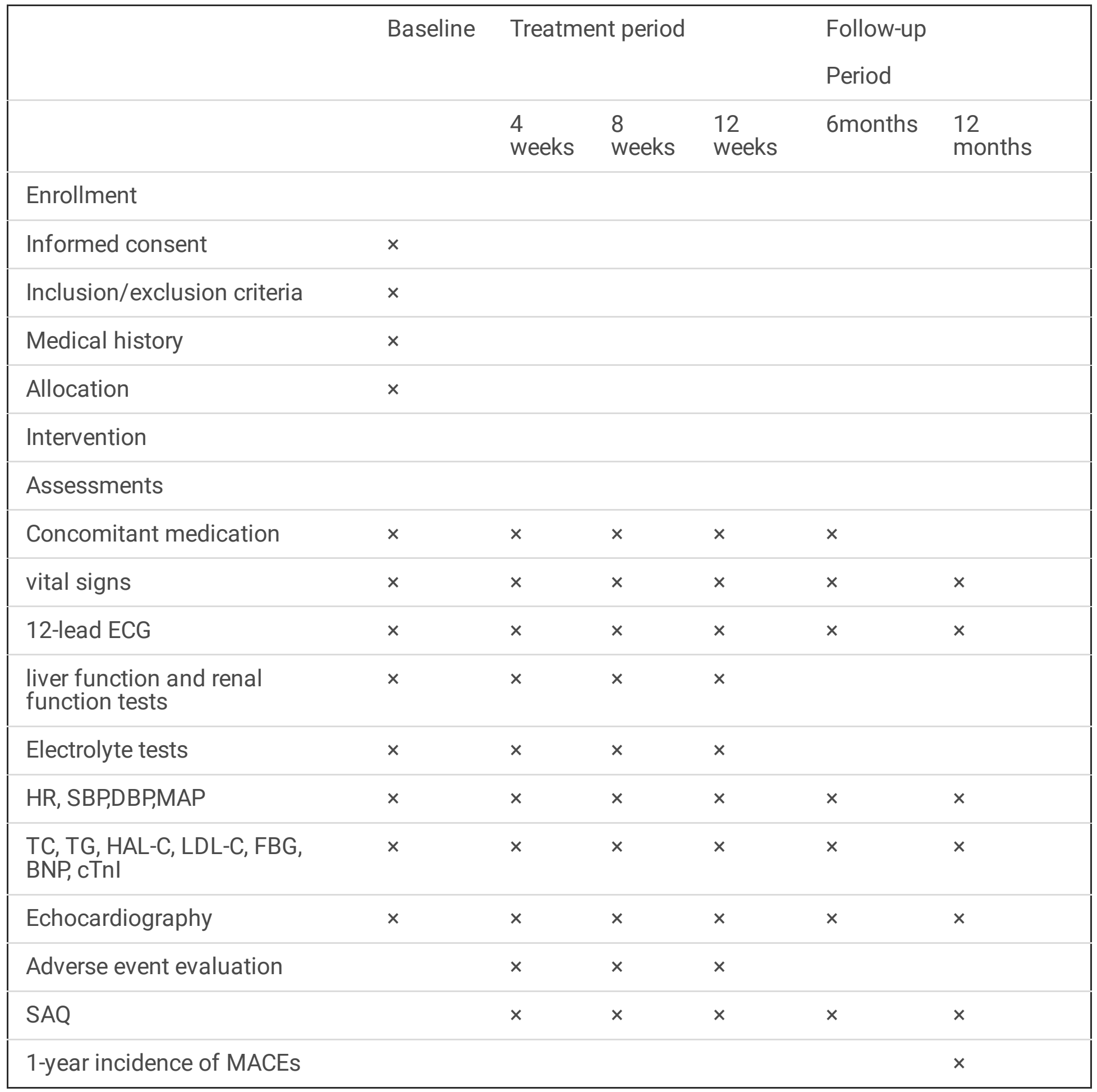

Abbreviations: TC: Total cholesterol; TG: Triglycerides; HDL-C: High density lipoprotein cholesterol; LDL-C: Low density lipoprotein cholesterol; FBG: Fasting blood glucose; SBP: Systolic blood pressure; DBP: Diastolic blood pressure; BNP: Brain natriuretic peptide; cTnl: cTroponin I.

\section{Figures}




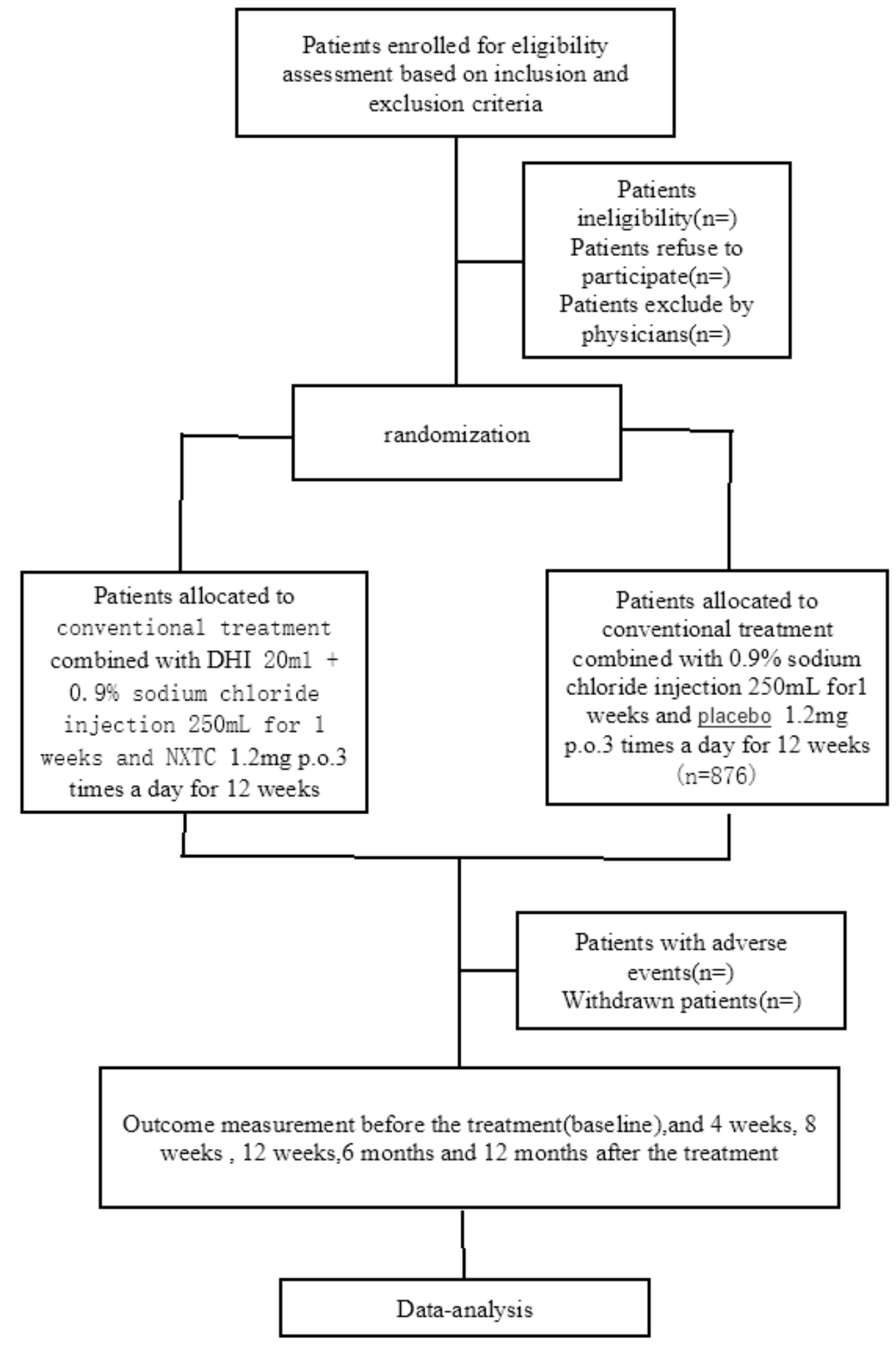

Figure 1

Study flow diagram 